IP Periodica Polytechnica

Mechanical Engineering

\author{
61(2), pp. 107-114, 2017 \\ https://doi.org/10.3311/PPme.9368 \\ Creative Commons Attribution (i)
}

RESEARCH ARTICLE

\section{Lagrangian Dynamics Analysis of a XY-Theta Parallel Robotic Machine Tool}

\author{
Javad Enferadi $^{1^{*}}$, Mohammad Tavakolian ${ }^{1}$
}

Received 24 April 2016; accepted after revision 14 December 2016

\begin{abstract}
Dynamics of a highly stiff parallel machine tool is the subject of this paper. High stiffness, good accuracy, relatively large workspace and free of singularities on the whole workspace makes the manipulator suitable for machining applications as an XY-Theta precision table. First, obtaining kinematics constraints, inverse kinematics analysis and velocity analysis are performed. Next, using six redundant generalized coordinates, we obtain Lagrangian of the manipulator. Also, a Lagrangian approach is proposed to obtain dynamics equations of the machine tool using three Lagrangian multipliers. This method allows elimination of constraint forces and moments at the joints from the motion equations. Dynamic equations of the manipulator are formed as inverse dynamics and direct dynamics problems. Finally, two examples are presented that confirms the obtained dynamics equations.
\end{abstract}

\section{Keywords}

parallel kinematics machine tool, Lagrangian dynamics, Lagrangian multipliers, inverse dynamics, forward dynamics

\footnotetext{
${ }^{1}$ Department of Mechanical Engineering, Mashhad Branch, Islamic Azad University, Mashhad, Iran

*Corresponding author, e-mail: Javadenferadi@gmail.com
}

\section{Introduction}

High precision is still one of the challenges when parallel kinematic machines (PKMs) are applied to advanced equipment. The parallel kinematic machines (PKMs) have significant advantages over conventional serial robots or mechanisms in terms of rigidity, dynamic load, operation speed, and accuracy. The PKMs should possess both the advantages of numerical control machines (higher stiffness, higher precision, and higher speed) and industrial robots (low cost, flexibility, ease of integration, and the operation in an open environment) [1-3].

A parallel manipulator typically consists of a moving platform and a fixed base that are connected together by several limbs. Because of the closed-loop architecture, not all joints can be independently actuated. In general, the number of actuated joints is equal to the number of degrees of freedom of the manipulator. Over the past decades, parallel mechanisms have received more and more attention from researchers and industries. They can be found in several practical applications, such as aircraft motion simulators [4], positional tracker [5-6], and micro-motion device [7-8]; as well as in the development of high-precision machine tools [9-14]. Ganesh, Rao and Darvekar presented a typical 3-DOF parallel kinematic machine (PKM) that provides translational motion along $\mathrm{X}, \mathrm{Y}$ and $\mathrm{Z}$ axes. This mechanism has three limbs each having an arm and a forearm. The joint arrangement is in such a way that the moving or tool platform maintains the same orientation in the entire workspace [15]. Joubair, Slamani and Bonev proposed a novel XYTheta precision table which can deliver accurate movements, is partially decoupled and has a relatively large workspace [16]. Recently the Exechon machine is introduced as an improvement over the Tricept design. The Exechon adopts a unique over constrained structure, and it has been improved based on the success of the Tricept [17].

In general, most existing PKMs can be classified into two main families. The first families of PKMs have fixed foot points and variable length struts and are generally called "Hexapods". The second families of PKMs have been more recently investigated. In this family, we can find the HEXAGLIDE (ETH Zurich Company) which features six parallel and six coplanar 
linear joints. The HexaM (Toyoda Company) is another example, with non-coplanar linear joints. A 3-axis translational version of the Hexaglide is the TRIGLIDE (Mikron company), which has three coplanar and parallel linear joints.

The development of a dynamical model is important in several different ways. First, a dynamical model can be used for computer simulation of a robotic system. Various manufacturing tasks can be examined without the need of a real system. Second, it can be used for the development of suitable control strategies. Third, the dynamic analysis reveals all the joint reaction forces and moments necessary for sizing the links, bearings and actuators. Dynamic analysis plays an important role in predicting the behavior of mechanical systems and achieving their best performances. There are two types of dynamical problems: (i) The direct dynamics problem aims to find the response of a robot arm corresponding to given applied moments and/or forces. That is, given the vector of joint moments or forces, it computes the resulting motion of the manipulator as a function of time. (ii) The inverse dynamics problem aims to find the actuator moments and/or forces required to generate a desired trajectory of the manipulator.

The inverse dynamics algorithm solves the following problem; given the desired trajectory of the end-effector as well as the mass distribution of each link, find actuator moments and/or forces required to generate this trajectory. The inverse dynamics analysis is needed for control: if one wants the robot to follow a specified trajectory, one has to convert the desired motion along that trajectory into joint forces that will generate this motion.

The direct dynamics algorithm solves the following problem; given the vector of initial actuated joint positions, vector of initial actuated joint velocities, applied actuated torques, applied external forces to end-effector, as well as the mass distribution of all links, find the resulting motion of the endeffector. The direct dynamics is used for simulation, and as feed-forward in the robot's motion controller, i.e., the direct dynamics calculates what the robot does when specific joint torques are applied, and under the assumption that all physical parameters in the dynamics model are accurate. Due to the closed-loop structure and kinematic constraints of PKMs, the derivation of dynamic equations is quite complicated. There are three main methods of formulation of the dynamical equations; namely, Newton-Euler laws, the Lagrangian formulation, the principle of virtual work [18-25] and Kane's method [26]. The Lagrangian formulation allows eliminating all of the reaction forces and moments at the beginning. Since a PKM has several closed-loop chains, it is a difficult task to derive the equations of motions in terms of a set of independent generalized coordinates. To simplify the problem, additional coordinates with a set of Lagrangian multipliers must be introduced. Also, Kane's method has two crucial considerations: (1) operational simplicity, meaning reduced labor in the derivation of the equations of motion either by hand or in terms of computer operations via symbol manipulation; and (2) simplicity of the final form of the equations, simplicity giving rise to reduction in computational time [26]. Both Lagrangian and Kane's methods can be used for holonomic and nonholonomic constraints, but Kane's method is better method for nonholonomic constraints.

$\mathrm{Wu}$, Li, and Wang optimized an asymmetrical hybrid machine tool based on dynamic isotropy [27]. Sung and Lu performed modeling and analysis of a four-half axis machine tool via modified Denavit-Hartenberg notation [28]. Mi, Yin, Sun and Wang analyzed effect of the joints as a significant parameter in the overall dynamic analysis of machine tools [29]. Kiran et. all performed inverse dynamics of three degrees of freedom (DOF) U-shaped planar parallel manipulator having three legs consisting of prismatic-prismatic-revolute (PPR) joint arrangement in which each leg has one active prismatic joint [30]. Jiang, Li and Wang proposed a novel planar 2-DOF parallel kinematic machine with kinematic redundancy and presented a method for redundant force optimization is presented to improve the precision of the machine. Also, they obtained dynamic model of the manipulator by the NewtonEuler method [31]. Wu, Chen, Li and Wang proposed a planar 2-DOF parallel manipulator with actuation redundancy and incorporated it into a 4-DOF hybrid machine tool. They also derived structural dynamics model of the manipulator to obtain its natural frequency [32].

\section{Structure description of the star-triangle planar parallel robot}

The use of long and slender legs in all of the mentioned designs causes vibration in high speed machining. To overcome this problem, Star-Triangle (ST) manipulator with virtually zero leg lengths is being considered here (see Fig. 1). High stiffness, good accuracy, relatively large workspace free of singularities [33] makes the manipulator suitable for machining applications. The manipulator has three degrees of freedom and can move the spindle of the machine in a plane. The machine can be augmented with a vertical motion as depicted in Fig. 2. This manipulator consists of a triangular base and a moving star that are connected via three PRP legs, in which $\mathbf{P}$ and $\mathbf{R}$ refer to prismatic and revolute pairs, respectively. The general model of this manipulator is depicted in Fig. 3, in which $P_{i}$ is the actuator. Moreover, $\mathrm{C}$ and $R_{i}$ are the end-effector (EE) and prismatic joint, respectively. Furthermore, $A_{i}$ is the joint with two degrees of freedom. If all leg lengths vanish, $\mathbf{r}_{i}=0$, then the structural stiffness of the manipulator increases, which make it suitable for machine tool applications (Figs. 1, 2 and 4). In Fig. 3, the geometric model of this manipulator is shown. 


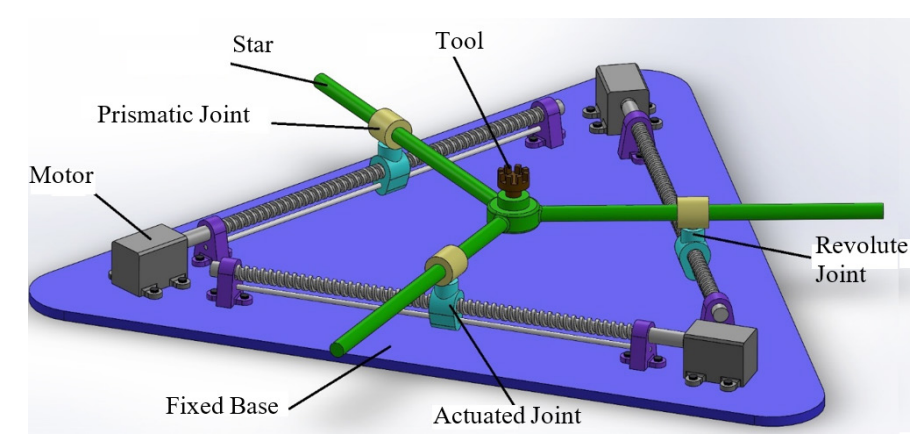

Fig. 1 Planar star-triangle (ST) manipulator

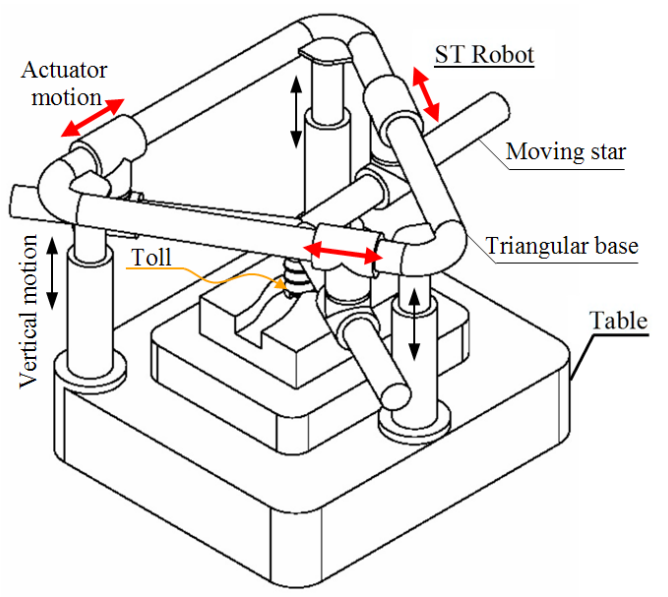

Fig. 2 The proposed ST Machine tool

\section{Kinematics Analysis}

\subsection{Kinematics constraints}

The Cartesian coordinates of point $\mathrm{C}$ of the $\mathrm{EE}$ and its orientation are given by $C(x, y)$ and $\varphi$, respectively (Fig. 4). The $L_{f}$ is the side length of the equilateral fixed base and the angles between three lines of the star is $120^{\circ}$. Once, the robot link parameters are fully defined, we can formulate the kinematics constraints for each limb. For this purpose, we obtain the position and orientation of the end-effector from each limb as (see Fig. 4)

$$
\begin{array}{ll}
\Lambda_{1}: & q_{1} \cos \theta_{1}+\rho_{1} \cos \varphi_{1}-x=0 \\
\Lambda_{2}: & q_{1} \sin \theta_{1}+\rho_{1} \sin \varphi_{1}-y=0 \\
\Lambda_{3}: & a_{1} \cos \theta_{1}+q_{2} \cos \theta_{2}+\rho_{2} \cos \varphi_{2}-x=0 \\
\Lambda_{4}: & a_{1} \sin \theta_{1}+q_{2} \sin \theta_{2}+\rho_{2} \sin \varphi_{2}-y=0 \\
\Lambda_{5}: & a_{3}-q_{3}+\rho_{3} \cos \varphi-x=0 \\
\Lambda_{6}: & \rho_{3} \sin \varphi-y=0 \\
\Lambda_{7}: & \alpha_{1}-\varphi+\varphi_{1}=0 \\
\Lambda_{8}: & \theta_{1}-\varphi_{1}+\beta_{2}+\alpha_{1}+\varphi_{2}-\theta_{2}-\pi=0 \\
\Lambda_{9}: & \theta_{2}-\varphi_{2}+\beta_{3}+\alpha_{3}+\varphi-2 \pi=0
\end{array}
$$

Where $\Lambda_{1}-\Lambda_{9}$ are constraints equations and $x, y, \varphi, \mathrm{q}_{1}, \mathrm{q}_{2}$ and $\mathrm{q}_{3}$ are generalized coordinates. Using combination and simplification of the above equations, we can reduce the constraint equations from (9) to (3) and rewrite as follows

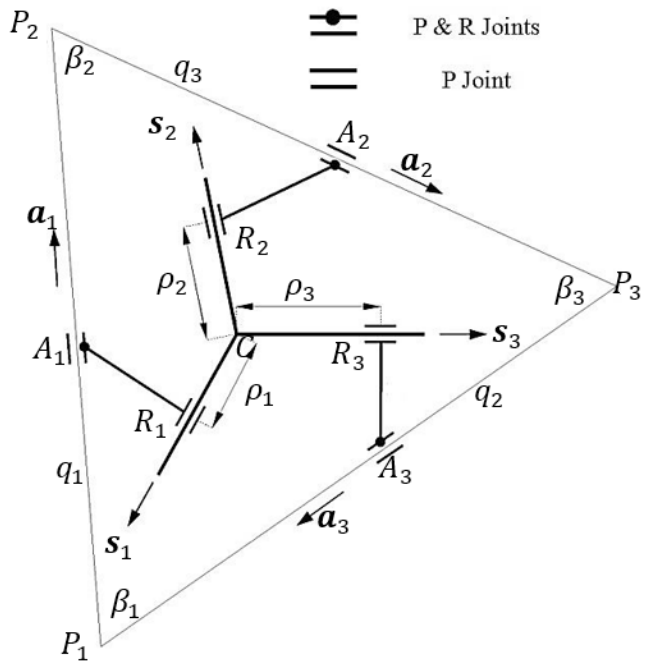

Fig. 3 General model of the ST manipulator

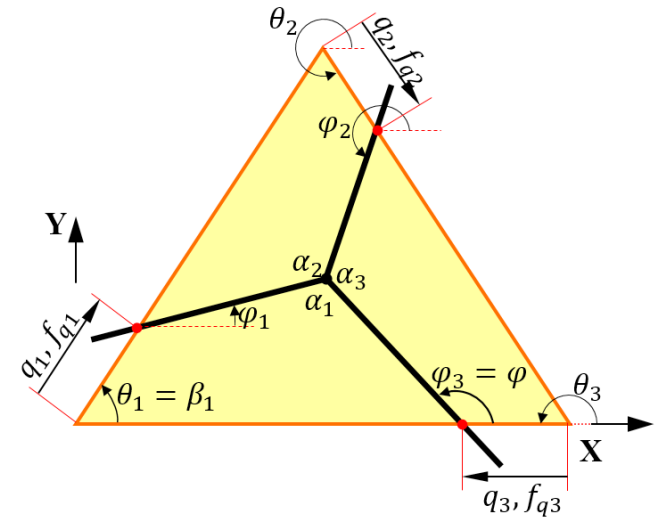

Fig. 4 Geometric model of the special ST manipulator

$$
\begin{array}{ll}
\Gamma_{1}: & \left(q_{1} \cos \theta_{1}-x\right)+\left(q_{1} \sin \theta_{1}-y\right) \cot \left(\alpha_{1}-\varphi\right)=0 \\
\Gamma_{2}: & q_{1} \cos \theta_{1}+q_{2} \cos \theta_{2}+ \\
& \left(y-q_{1} \sin \theta_{1}+q_{2} \sin \theta_{2}\right) \cot \left(\alpha_{3}+\varphi\right)-x=0 \\
\Gamma_{3}: & a_{3}-q_{3}+y \cot \varphi-x=0
\end{array}
$$

\subsection{Inverse kinematics problem}

The inverse kinematics problem of a manipulator is the search for the parameters of the motions of all actuated kinematic pairs in the sub-chains that is based on the desired position and orientation of the moving platform. In this paper, a solution of the inverse position problem is required for the dynamics analysis of the manipulator. Referring to Fig. 4, the closure equation for each limb can be written as

$$
\mathbf{c}=\mathbf{P}_{i}+q_{i} \boldsymbol{a}_{i}-\rho_{i} \mathbf{s}_{i}
$$

Multiplying both sides of the above equation by $\boldsymbol{a}_{i}^{T} \mathbf{E}$, yields

$$
\rho_{i}=-\boldsymbol{a}_{i}^{T} \mathbf{E}\left(\mathbf{c}-\mathbf{P}_{i}\right) /\left(\boldsymbol{a}_{i}^{T} \mathbf{E s}_{i}\right)
$$

Where $\mathbf{E}_{2 \times 2}$ is an orthogonal matrix that rotates any vector in a plane through an angle of $90^{\circ}$ counterclockwise. Now, we multiply both sides of the Eq. (3) by $\mathbf{s}_{i}^{T} \mathbf{E}$, to obtain $q_{i}$ as 


$$
q_{i}=\mathbf{s}_{i}^{T} \mathbf{E}\left(\mathbf{c}-\mathbf{P}_{i}\right) /\left(\mathbf{s}_{i}^{T} \mathbf{E} \boldsymbol{a}_{i}\right)
$$

\subsection{Velocity analysis}

The differential kinematics relations pertaining to parallel manipulators take on the form

$$
\mathbf{J} \dot{\mathbf{q}}+\mathbf{K} \dot{\mathbf{X}}=0
$$

where $\mathbf{J}$ and $\mathbf{K}$ are the two Jacobian matrices of the manipulator at hand. Moreover, $\dot{\mathbf{q}}$ is the vector of joint rates and $\dot{\mathbf{X}}$ is the twist array and is defined as

$$
\dot{\mathbf{X}}=[\omega \dot{\mathbf{c}}]^{T}=\left[\begin{array}{lll}
\dot{\varphi} & \dot{x} & \dot{y}
\end{array}\right]^{T}
$$

in which, $\omega$ is the scalar angular velocity of the moving star and $\dot{\mathbf{c}}$ is the two dimensional velocity vector of the operation point C. According to Fig. 3, the velocity $\dot{\mathbf{c}}$ can be written for the $\mathrm{i}^{\text {th }}$ leg as

$$
\dot{\mathbf{c}}=\mathbf{V}_{A i}+\left(\mathbf{V}_{R i}-\mathbf{V}_{A i}\right)+\left(\dot{\mathbf{c}}-\mathbf{V}_{R i}\right)
$$

where $\mathbf{V}_{A i}$ and $\mathbf{V}_{R i}$ are the absolute velocity vectors of points $A_{i}$ and $R_{i}$, respectively. Moreover, we have

$$
\mathbf{V}_{A i}=\dot{q}_{i} \boldsymbol{a}_{i}
$$

where $\boldsymbol{a}_{i}$ is the unit vector directed from $\mathrm{P}_{i}$ to $A_{i}$, and $\dot{\mathrm{q}}_{i}$ is the rate of $\mathrm{i}^{\text {th }}$ actuator. Furthermore, we have

$$
\mathbf{V}_{R i}-\mathbf{V}_{A i}=\dot{\varphi} \mathbf{E} \mathbf{r}_{i}
$$

Moreover, $\mathbf{r}_{i}$ is the vector from $A_{i}$ to $R_{i}$ and is assumed to have zero length for the manipulator at hand. Therefore, Eq. (10) leads to

$$
\mathbf{V}_{R i}-\mathbf{V}_{A i}=0
$$

Finally, we have

$$
\dot{\mathbf{c}}-\mathbf{V}_{R i}=\dot{\rho}_{i} \mathbf{s}_{i}-\rho_{i} \omega \mathbf{E} \mathbf{s}_{i}
$$

where $\rho_{i}$ is the distance from $R_{i}$ to $\mathrm{C}$ and $\mathbf{s}_{i}$ is the unit vector representing the direction of the third prismatic joint. Substituting the corresponding values from Eqs. (9) to (12) into (8), leads to

$$
\dot{q}_{i} \boldsymbol{a}_{i}+\dot{\rho}_{i} \mathbf{s}_{i}-\omega \rho_{i} \mathbf{E s}_{i}-\dot{\mathbf{c}}=0
$$

Upon multiplication of the two sides of the above equation by $\mathbf{s}_{i}^{T} \mathbf{E}$, we can obtain

$$
\dot{q}_{i} \mathbf{s}_{i}^{T} \mathbf{E} \boldsymbol{a}_{i}+\omega \rho_{i} \mathbf{s}_{i}^{T} \mathbf{s}_{i}-\mathbf{s}_{i}^{T} \mathbf{E} \dot{\mathbf{c}}=0
$$

Equation (14) can be written for $\mathrm{i}=1,2,3$, which produces Eq. (6), in which the Jacobian matrices $\mathbf{J}$ and $\mathbf{K}$ are given as

$$
\mathbf{K}=\left[\begin{array}{cc}
\rho_{1} & -\mathbf{s}_{1}^{T} \mathbf{E} \\
\rho_{2} & -\mathbf{s}_{2}^{T} \mathbf{E} \\
\rho_{3} & -\mathbf{s}_{3}^{T} \mathbf{E}
\end{array}\right]
$$

$$
\mathbf{J}=\left[\begin{array}{ccc}
\mathbf{s}_{1}^{T} \mathbf{E} \boldsymbol{a}_{1} & 0 & 0 \\
0 & \mathbf{s}_{2}^{T} \mathbf{E} \boldsymbol{a}_{2} & 0 \\
0 & 0 & \mathbf{s}_{3}^{T} \mathbf{E} \boldsymbol{a}_{3}
\end{array}\right]
$$

Furthermore, we can rewrite Eq. (6) as inverse velocity and direct velocity as follows

$$
\begin{gathered}
\dot{\mathbf{q}}=-\mathbf{J}^{-1} \mathbf{K} \dot{\mathbf{X}}=\mathbf{H} \dot{\mathbf{X}} \\
\dot{\mathbf{X}}=-\mathbf{K}^{-1} \mathbf{J} \dot{\mathbf{q}}=\mathbf{H}^{-1} \dot{\mathbf{q}}=\mathbf{G} \dot{\mathbf{q}}
\end{gathered}
$$

We use these forms for inverse and direct dynamics analyses.

\section{Energy and Lagrangian of the ST robot}

Due to the ST manipulator works in a horizontal plane, its the potential energy is zero. Therefore, the energy of the ST manipulator divided into kinetic energies of the three actuators, the three intermediate links and the traveling plate (Star). Now, we will compute the energy of each part individually.

\subsection{Kinetic energy of the traveling Plate (star)}

The kinetic energy of the traveling plate, $T_{p}$, can be divided into two terms; translational and rotational kinetic energies. Translational kinetic energy, $T_{p}^{t}$, is the energy of traveling plate's mass center when one considers that the entire mass is concentrated there. The rotational kinetic energy, $T_{p}^{r}$, is due to rotation of the traveling plate around an axis that is perpendicular to the horizontal plane. These kinetic energies can be express as

$$
T_{p}=T_{p}^{t}+T_{p}^{r}=\frac{1}{2} m_{p}\left(\dot{x}^{2}+\dot{y}^{2}\right)+\frac{1}{2} I_{p} \dot{\phi}^{2}
$$

Where $m_{p}$ is mass of the star platform and $I_{p}$ is inertia of the star around an axis that pass from its mass center and is perpendicular to the horizontal plane.

\subsection{Kinetic energy of the intermediate link}

As the case for the traveling plate kinetic energies of the intermediate links are divided into translational $\left(T_{i l}^{t}\right)$ and rotational $\left(T_{i l}^{r}\right)$ kinetic energies and calculated as

$$
\begin{aligned}
T_{i l} & =T_{i l}^{t}+T_{i l}^{r}=\sum_{i=1}^{3} \frac{1}{2} m_{i l} \dot{q}_{i}^{2}+\sum_{i=1}^{3} \frac{1}{2} I_{i i} \dot{\beta}_{i}^{2} \\
& =\frac{1}{2} m_{i l}\left(\dot{q}_{1}^{2}+\dot{q}_{2}^{2}+\dot{q}_{3}^{2}\right)+\frac{3}{2} I_{i i} \dot{\phi}^{2}
\end{aligned}
$$

Where $m_{i l}$ is mass of the each intermediate link and $I_{i l}$ is inertia of the intermediate link around an axis that pass from mass center of the intermediate link and is perpendicular to the horizontal plane. 


\subsection{Kinetic energy of the actuated link}

The actuated links have only translational kinetic energy that is expressed as

$$
T_{a}=\sum_{i=1}^{3} \frac{1}{2} m_{a} \dot{q}_{i}^{2}=\frac{1}{2} m_{a}\left(\dot{q}_{1}^{2}+\dot{q}_{2}^{2}+\dot{q}_{3}^{2}\right)
$$

Where $m_{s}$ is mass of the each actuated link.

\subsection{Lagrangian of the ST manipulator}

Since the potential energy of the whole system is zero, Lagrangian of the whole system is a summation of Lagrangians of the actuated links, the intermediated links and the star. Therefore, we can write Lagrangian as follows

$$
\begin{aligned}
L & =\frac{1}{2} m_{a}\left(\dot{q}_{1}^{2}+\dot{q}_{2}^{2}+\dot{q}_{3}^{2}\right)+\frac{1}{2} m_{i l}\left(\dot{q}_{1}^{2}+\dot{q}_{2}^{2}+\dot{q}_{3}^{2}\right) \\
& +\frac{3}{2} I_{i l} \dot{\phi}^{2}+\frac{1}{2} m_{p}\left(\dot{x}^{2}+\dot{y}^{2}\right)+\frac{1}{2} I_{p} \dot{\phi}^{2}
\end{aligned}
$$

\section{Dynamics Analysis}

As stated before, both Lagrangian and Kane's methods can be used for holonomic and nonholonomic constraints. However, because of simplicity, the Kane's method is better method for nonholonomic constraints. Since the ST manipulator is planar and the holonomic constraints are applied to obtain equations of motion, we employ the Lagrangian method. To evaluate Lagrangian of the planar ST manipulator, the velocities of the actuated joints, intermediate links and traveling plate (Star) are computed. The Lagrangian of the planar ST manipulator is related to generalized velocities of actuated joints and the velocity of the traveling plate. There are two ways to compute the dynamical model of the system; the first way is to express Lagrangian of the ST manipulator only as a function of generalized coordinates on the traveling plate $(\varphi, x, y)$, or generalized coordinates on the actuated link $\left(q_{1}, q_{2}, q_{3}\right)$, which is complicated because the relationship between the actuated joints and the traveling plate (position and orientation) is nonlinear (see Eq. (2)). In the second way, Lagrangian of the ST manipulator will be written as a function of both actuated, $\left(q_{1}, q_{2}, q_{3}\right)$, and redundant generalized coordinates $(\varphi, x, y)$ which are related to each other through $\Gamma_{k}$ constraint (for $\mathrm{k}=1,2,3$ ) denoting the $\mathrm{k}^{\text {th }}$ constraint function (Eq. (2)). Therefore, we can now write Lagrangian equation based on the second way as

$$
\frac{d}{d t}\left(\frac{\partial L}{\partial \dot{q}_{j}}\right)-\frac{\partial L}{\partial q_{j}}=Q_{j}+\sum_{k=1}^{m} \lambda_{k} \frac{\partial \Gamma_{k}}{\partial q_{j}} j=1,2, \ldots, 6
$$

Where $k$ is the number of constraint functions and $\lambda_{k}$ are the Lagrangian multiplier. As stated before, Lagrangian of the ST manipulator is a function of a vector which is a vector containing all the actuated joints and the traveling plate (Star). Using Lagrangian, we can drive dynamics equations of the ST manipulator as
I: $\left(m_{p}+m_{i l}\right) \ddot{q}_{1}=f_{q_{1}}+\lambda_{1}\left(\cos \theta_{1}+\sin \theta_{1} \cot \left(\alpha_{1}-\varphi\right)\right)$

II: $\left(m_{p}+m_{i l}\right) \ddot{q}_{2}=f_{q_{2}}+\lambda_{2}\left(\cos \theta_{2}+\sin \theta_{2} \cot \left(\alpha_{2}+\varphi\right)\right)$

III: $\left(m_{p}+m_{i l}\right) \ddot{q}_{3}=f_{q_{3}}-\lambda_{3}$

IV: $m_{p} \ddot{x}=f_{x}-\lambda_{1}-\lambda_{2}-\lambda_{3}$

$\mathrm{V}: m_{p} \ddot{y}=f_{y}-\lambda_{1} \cot \left(\alpha_{1}-\varphi\right)+\lambda_{2} \cot \left(\alpha_{2}+\varphi\right)+\lambda_{3} \cot \varphi$

VI: $\left(I_{p}+3 I_{i l}\right) \ddot{\varphi}=n_{\text {ext. }}+\lambda_{1}\left(q_{1} \sin \theta_{1}-y\right)\left(1+\cot ^{2}\left(\alpha_{1}-\varphi\right)\right)$

$+\lambda_{2}\left(q_{1} \sin \theta_{1}-y-q_{2} \sin \theta_{2}\right)\left(1+\cot ^{2}\left(\alpha_{3}+\varphi\right)\right)-\lambda_{3} y\left(1+\cot ^{2} \varphi\right)$

Now, the six above equations can be written as a matrix form as follows

$$
\left[\begin{array}{cc}
\mathbf{M} & 0 \\
0 & \mathbf{N}
\end{array}\right]_{6 \times 6}\left[\begin{array}{c}
\ddot{\mathbf{q}} \\
\ddot{\mathbf{X}}
\end{array}\right]_{6 \times 1}=\left[\begin{array}{c}
\mathbf{A} \\
\mathbf{B}
\end{array}\right]_{6 \times 3}\left[\begin{array}{c}
\lambda_{1} \\
\lambda_{2} \\
\lambda_{3}
\end{array}\right]_{3 \times 1}+\left[\begin{array}{c}
\boldsymbol{f}_{q} \\
\boldsymbol{f}_{\text {ext. }}
\end{array}\right]_{6 \times 1}
$$

where $\boldsymbol{f}_{q}=\left[\begin{array}{lll}f_{q_{1}} & f_{q_{2}} & f_{q_{3}}\end{array}\right]^{T}$ and $\boldsymbol{f}_{\text {ext. }}=\left[\begin{array}{lll}n_{\text {ext. }} & f_{x} & f_{y}\end{array}\right]^{T}$ are the actuated forces and the applied external wrench to the endeffector, respectively, and

$$
\begin{gathered}
\mathbf{M}=\left[\begin{array}{ccc}
m_{s}+m_{l} & 0 & 0 \\
0 & m_{s}+m_{l} & 0 \\
0 & 0 & m_{s}+m_{l}
\end{array}\right] \\
\mathbf{N}=\left[\begin{array}{ccc}
m_{p} & 0 & 0 \\
0 & n_{p} & 0 \\
0 & 0 & I_{p}+3 I_{l}
\end{array}\right] \\
\mathbf{A}=\left[\begin{array}{ccc}
a_{11} & 0 & 0 \\
0 & a_{22} & 0 \\
0 & 0 & a_{33}
\end{array}\right] \\
\mathbf{B}=\left[\begin{array}{ccc}
-1 & -1 & -1 \\
b_{21} & b_{22} & b_{23} \\
b_{31} & b_{32} & b_{33}
\end{array}\right]
\end{gathered}
$$

In which,

$$
\begin{aligned}
& a_{11}=\cos \theta_{1}+\sin \theta_{1} \cot \left(\alpha_{1}-\varphi\right) \\
& a_{22}=\cos \theta_{3}+\sin \theta_{3} \cot \left(\alpha_{3}+\varphi\right) \\
& a_{33}=-1 \\
& b_{21}=-\cot \left(\alpha_{1}-\varphi\right) \\
& b_{22}=\cot \left(\alpha_{3}+\varphi\right) \\
& b_{23}=\cot \varphi \\
& b_{31}=\left(q_{1} \sin \theta_{1}-y_{c}\right)\left(1+\cot ^{2}\left(\alpha_{1}-\varphi\right)\right) \\
& b_{32}=\left(a_{1} \sin \theta_{1}-y_{c}-q_{2} \sin _{3}\right)\left(1+\cot ^{2}\left(\alpha_{3}+\varphi\right)\right) \\
& b_{33}=-y_{c}\left(1+\cot ^{2} \varphi\right)
\end{aligned}
$$

Now, we multiply both sides of the Eq. (25) by $\mathbf{W}=\mathbf{B} \mathbf{A}^{-1}$ and rewrite it as 


$$
\mathbf{M} \ddot{\mathbf{X}}-\mathbf{W N} \ddot{\mathbf{q}}+\mathbf{W} \boldsymbol{f}_{q}=\boldsymbol{f}_{\text {ext }}
$$

On the other hand, using Eqs. (16) and (17), we can write

$$
\begin{aligned}
& \ddot{\boldsymbol{q}}=\mathbf{H} \ddot{\mathbf{X}}+\dot{\mathbf{H}} \dot{\mathbf{X}} \\
& \ddot{\boldsymbol{X}}=\mathbf{G} \ddot{\mathbf{q}}+\dot{\mathbf{G}} \dot{\mathbf{q}}
\end{aligned}
$$

Now, substituting the above equations into (31), yields

$$
(\mathbf{M}-\mathbf{W N H}) \ddot{\mathbf{X}}-\mathbf{W N} \dot{\mathbf{H}} \dot{\mathbf{X}}+\mathbf{W} \boldsymbol{f}_{q}=\boldsymbol{f}_{\text {ext. }}
$$

$$
(\mathbf{M G}-\mathbf{W N}) \ddot{\mathbf{q}}+\mathbf{M} \dot{\mathbf{G}} \dot{\mathbf{q}}+\mathbf{W} \boldsymbol{f}_{q}=\boldsymbol{f}_{\text {ext }}
$$

Equation (33) is called direct dynamic equation form of the ST manipulator. In this form, input forces by the actuators, $\boldsymbol{f}_{q}$, and the applied external wrench to the end-effector, $f_{\text {ext. }}$, are known. We will compute trajectory of the end-effector. Furthermore, Eq. (34) is as an inverse dynamic equation form. For the inverse dynamics problem, a desired path of the star is given and the problem is determining the input torques required to produce the motion.

\section{Case studies}

Based on the previous section, a computer program is developed using MATLAB software. Two separate paths for the ST planar robot are considered. In the first path, Point $\mathrm{C}$ moves along a vertical line and in the second path, point $\mathrm{C}$ moves along a circular path. The parametric equations for these paths are given as

First path: vertical line

$$
C=\left[\begin{array}{ll}
x & y
\end{array}\right]^{T}=\left[\begin{array}{ll}
(a / 2) & (a / 2)+(a / 5) \cos \pi t / 4
\end{array}\right]^{T}, t \in\left[\begin{array}{ll}
0 & 8
\end{array}\right]
$$

Second path: circular path

$$
C=\left[\begin{array}{ll}
(a / 2)+(a / 8) \cos \pi t \quad(a / 2)+(a / 8) \sin \pi t
\end{array}\right]^{T}, t \in\left[\begin{array}{ll}
0 & 2
\end{array}\right]
$$

Moreover, the direction of the star is chosen to be $\varphi=\pi / 2$. This value will ensure maximum workspace [33] and thus the robot can travel its entire triangular base. Therefore, we will keeping this value constant during our simulations. The results are verified in two ways. First, using inverse dynamics problem, a trajectory for the MSS is supplied and required motor torques as well as the linear position of the actuators as a function of time are calculated for each path (inverse dynamics). Then, the initial conditions (position and velocity) and the calculated torques from the inverse dynamics problem are applied to the actuators for obtaining the path of the star. If the output of the inverse dynamics problem, is supplied to the direct dynamics problem, then the same trajectory for the star must be obtained. In this case, the obtained Eqs. (33) and (34) for the dynamics analysis are verified. Other parameters are assumed as follows

$$
\begin{array}{llc}
a=a_{i}=0.5 \mathrm{~m} & \alpha_{i}=120^{\circ} & \theta_{i}=60^{\circ} \\
m_{a}=2 \mathrm{~kg} & m_{i l}=3 \mathrm{~kg} & m_{p}=2 \mathrm{~kg} \\
I_{i l}=37.5 \mathrm{~kg} \cdot \mathrm{m}^{2} & I_{p}=25 \mathrm{~kg} \cdot \mathrm{m}^{2} \\
\boldsymbol{f}_{\text {ext. }}=\mathbf{0} & &
\end{array}
$$

Simulation of the linear and circular paths for both inverse and direct dynamics problems is shown in Figs. 5 to 8. As shown, the results confirm each other. Therefore, the obtained formulations for a dynamical model of the ST manipulator are correct.

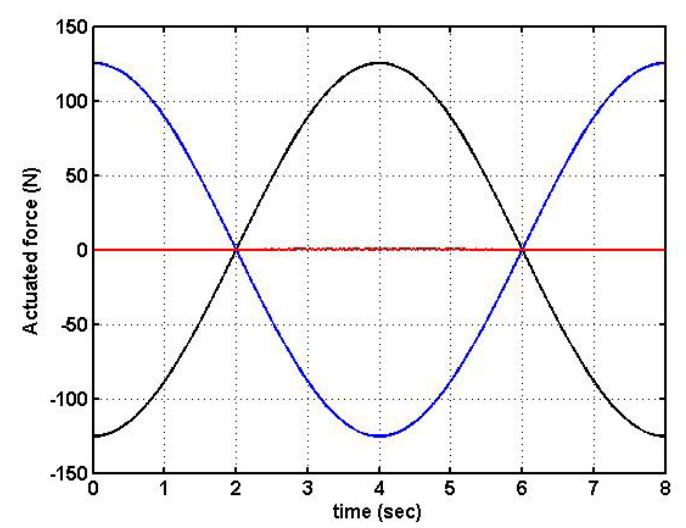

Fig. 5 Calculated actuator forces for the linear path (inverse dynamics)

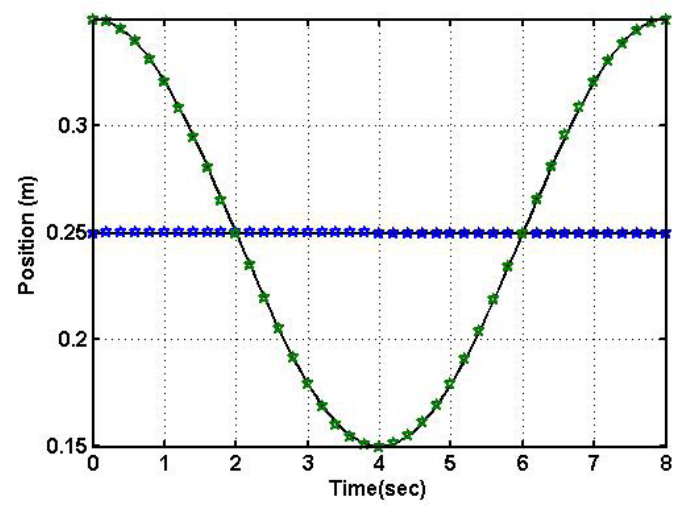

Fig. 6 Path of the star due to applied forces to the actuators according to Fig. 5 (direct dynamics)

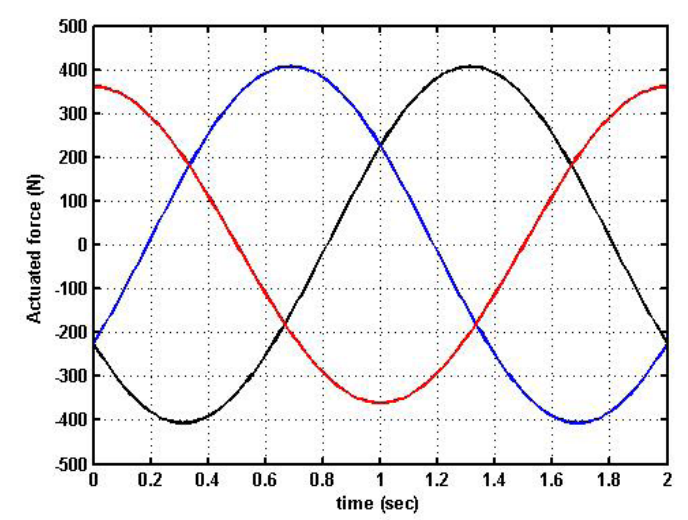

Fig. 7 Calculated actuator forces for the circular path (inverse dynamics) 


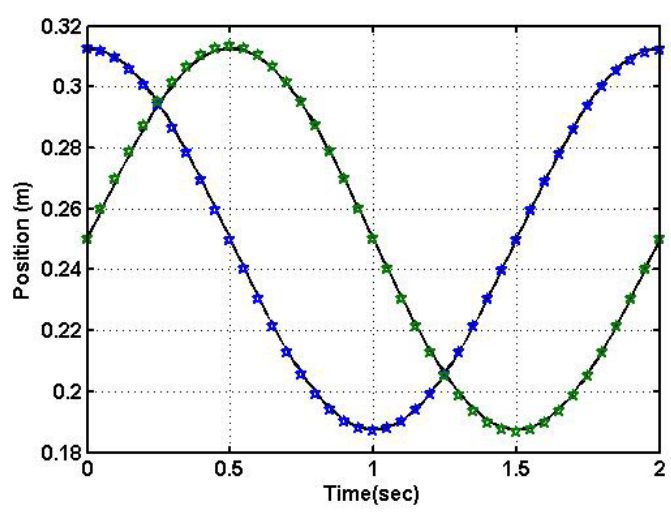

Fig. 8 Path of the star due to applied forces to the actuators according to Fig. 7 (direct dynamics)

\section{Conclusion}

A planar parallel robotic machine tool with high stiffness was considered. good accuracy, relatively large workspace free of singularities made the manipulator suitable for machining applications. First, obtaining kinematics constraints, inverse kinematics analysis and velocity analysis were performed. Next, the six redundant generalized coordinates and three Lagrangian multipliers were introduced. Using a Lagrangian approach was obtained dynamics equations of the machine tool. The Lagrangian method was allowed elimination of constraint forces and moments at the joints from motion equations. Dynamic equations of the manipulator were formed for inverse and direct dynamics analyses. Finally, two examples were presented that verified the obtained dynamics equations.

\section{References}

[1] Olazagoitia, J. L., Wyatt, S. "New PKM Tricept T9000 and its application to flexible manufacturing at aerospace industry." SAE Technical Paper 2007-01-3820, 2007. https://doi.org/10.4271/2007-01-3820

[2] Bi, Z. M., Jin, Y. "Kinematic modeling of Exechon parallel kinematic machine." Robotics and Computer-Integrated Manufacturing. 27(1), pp. 186-193. 2011. https://doi.org/10.1016/j.rcim.2010.07.006

[3] Li, M., Huang, T., Mei, J., Zhao, X., Chetwynd, D. G., Hu, S. J. "Dynamic formulation and performance comparison of the 3-DOF modules of two reconfigurable PKMs--The Tricept and The TriVariant." Journal of Mechanical Design. 127(6), pp. 1129-1136. 2005. https://doi.org/10.1115/1.1992511

[4] Dasgupta, B., Mruthyunjaya, T. S. "The Stewart platform manipulator: a review." Mechanism and Machine Theory. 35(1), pp. 15-40. 2000. https://doi.org/10.1016/S0094-114X(99)00006-3

[5] Dunlop, G. R., Jones, T. P. "Position analysis of a two-DOF parallel mechanism- the Canterbury tracker." Mechanism and Machine Theory. 34(4), pp. 599-614. 1999. https://doi.org/10.1016/S0094-114X(98)00020-2

[6] Visa, I., Comsit, M. "Tracking systems for solar energy conversion devices." In: 14th International Sonnenforum. Freiburg, Germany. Jun. 2023, 2004. pp. 783-788.
[7] Kang, D. S., Seo, T. W., Kim, J. "Development and kinematic calibration for measurement structure of a micro parallel mechanism platform." Journal of Mechanical Science and Technology. 22(4), pp. 746-754. 2008. https://doi.org/10.1007/s12206-008-0107-4

[8] Lee, K. M., Arjunan, S. "A three-degrees-of-freedom micro motion in parallel actuated manipulator." IEEE Tranactions on Robotics and Automation. 7(5), pp. 634-641. 1991.

[9] Fedewa, D., Mehrabi, M. D., Kota, S., Orlandea, N., Gopalakrishnan, V. "Parallel structures and their applications in reconfigurable machining systems." In: Proceedings of the 2000 parallel kinematics machines international conference, Ann-Arbor, MI, Sep. 13-15, 2000, pp. 87-97.

[10] Huang, T., Li, Z. X., Li, M., Chetwynd, D., Gosselin, C. M. "Conceptual design and dimensional synthesis of a novel 2-DOF translational parallel robot for pick-and-place operations." Journal of Mechanical Design. 126(3), pp. 449-455, 2004.

https://doi.org/10.1115/1.1711822

[11] Wenger, P., Chablat, D. "Design of a three-axis isotropic parallel manipulator for machining applications: The Orthoglide." In: Proceedings of the workshop on fundamental issues and future research directions for parallel mechanisms and manipulators. (Gosselin, C. M., Ebert-Uphoff, I. (ed.), Quebec City, Quebec, Canada, Oct. 3-4, 2002. pp. 16-24.

[12] Fan, K. C., Wang, H., Zhao, J. W., Chang, T. H. "Sensitivity analysis of the 3-PRS parallel kinematic spindle platform of a serial-parallel machine tool." International Journal of Machine Tools and Manufacture. 43(15), pp. 1561-1569. 2003. https://doi.org/10.1016/S0890-6955(03)00202-5

[13] Enferadi, J., Mohammadi Daniali, H. R., Hojjati, M. "Isotropic design of a planar parallel manipulators for machining applications." Journal of the Engineering Faculty of Ferdowsi University. 15, pp. 125-137. 2002.

[14] Tlustry, J., Ziegart, J., Ridgeway, S. "Fundamental comparison of the use of serial and parallel kinematics for rmachine tools." CIRP Annals Manufacturing Technology. 48(1), pp. 351-356. 1999. https://doi.org/10.1016/S0007-8506(07)63200-4

[15] Ganesh, S. S., Koteswara Rao, A. B., Darvekar, S. "Multi-objective optimization of a 3-DOF translational parallel kinematic machine." Journal of Mechanical Science and Technology. 27(12), pp. 3797-3804. 2013. https://doi.org/10.1007/s12206-013-0957-2

[16] Joubair, A., Slamani, M., Bonev, I. A. "A novel XY-Theta precision table and a geometric procedure for its kinematic calibration." Robotics and Computer-Integrated Manufacturing. 28(1), pp. 57-65. 2012. https://doi.org/10.1016/j.rcim.2011.06.006

[17] Bi, Z. M., Jin, Y. "Kinematic modeling of Exechon parallel kinematic machine." Journal of Robotics and Computer-Integrated Manufacturing. 27(1), pp. 186-193. 2011.

https://doi.org/10.1016/j.rcim.2010.07.006

[18] Koteswara Rao, A. B., Saha, S. K., Rao, P. V. M. "Dynamics modelling of hexa-slides using the decoupled natural orthogonal complement matrices." Multibody System Dynamics. 15(2), pp. 159-180. 2006. https://doi.org/10.1007/s11044-005-9003-1

[19] Staicu, S., Zhang, D. "A novel dynamic modeling approach for parallel mechanisms analysis." Robotics and Computer-Integrated Manufacturing. 24(1), pp. 167-172. 2008. https://doi.org/10.1016/j.rcim.2006.09.001

[20] Staicu, S. "Inverse dynamics of the 3-PRR planar parallel robot." Robotics and Autonomous Systems. 57(5), pp. 556-563. 2009. https://doi.org/10.1016/j.robot.2008.09.005

[21] Staicu, S. "Dynamics analysis of the Star parallel manipulator." Robotics and Autonomous Systems. 57(11), pp. 1057-1064. 2009. https://doi.org/10.1016/j.robot.2009.07.005 
[22] Enferadi, J., Tootoonchi, A. A. "Inverse dynamics analysis of a general spherical star-triangle parallel manipulator using principle of virtual work." Nonlinear Dynamics. 61(3), pp. 419-434. 2010.

https://doi.org/10.1007/s11071-010-9659-9

[23] Enferadi, J., Tootoonchi, A. A. "A virtual work based algorithm for solving direct dynamics problem of a 3-RRP spherical parallel manipulator." Journal of Intelligent and Robotics Systems. 63(1), pp. 25-49. 2011. https://doi.org/10.1007/s10846-010-9469-9

[24] Enferadi, J., Tootoonchi, A. A. "A novel spherical parallel manipulator: forward position problem, singularity analysis, and isotropy design." Robotica. 27(5), pp. 663-676. 2009. https://doi.org/10.1017/S0263574708005031

[25] Staicu, S. "Recursive modeling in dynamics of Agile Wrist spherical parallel robot." Robotics and Computer-Integrated Manufacturing. 25(2), pp. 409-416. 2009. https://doi.org/10.1016/j.rcim.2008.02.001

[26] Banerjee, A. K. "Flexible Multibody Dynamics: Efficient Formulations and Applications." John Wiley \& Sons, 2016.

[27] Wu, J., Li, T., Wang, L. "Counterweight optimization of an asymmetrical hybrid machine tool based on dynamic isotropy." Journal of Mechanical Science and Technology. 27(7), pp. 1915-1922. 2013. https://doi.org/10.1007/s12206-013-0503-2

[28] Sung, C. K., Lu, C. H. "Modeling/analysis of four-half axis machine tool via modified denavit-hartenberg notation." Journal of Mechanical Science and Technology. 28(12), pp. 5135-5142. 2014.

https://doi.org/10.1007/s12206-014-1136-9
[29] Mi, L., Yin, G. F., Sun, M., Wang, X. "Effects of pre-loads on joints on dynamic stiffness of a whole machine tool structure." Journal of Mechanical Science and Technology. 26(2), pp. 495-508. 2012.

https://doi.org/10.1007/s12206-011-1033-4

[30] Singh, Y., Vinoth, V., Kiran, Y. R., Mohanta, J. K., Mohan, S. "Inverse dynamics and control of a 3-DOF planar parallel (U-shaped 3-PPR) manipulator." Robotics and Computer-Integrated Manufacturing. 34, pp. 164-179. 2015.

https://doi.org/10.1016/j.rcim.2015.02.007

[31] Jiang, Y., Li. T., Wang, L. "Dynamic modeling and redundant force optimization of a 2-DOF parallel kinematic machine with kinematic redundancy." Robotics and Computer-Integrated Manufacturing. 32, pp. 1-10. 2015. https://doi.org/10.1016/j.rcim.2014.08.001

[32] Wu, J., Chen, X., Li, T., Wang, L. "Optimal design of a 2-DOF parallel manipulator with actuation redundancy considering kinematics and natural frequency." Robotics and Computer-Integrated Manufacturing. 29(1), pp. 80-85. 2013.

https://doi.org/10.1016/j.rcim.2012.07.005

[33] Zarkandi, S. "Kinematics of a star-triangle planar parallel manipulator." Journal of Mechanical Science and Technology. 25(1), pp. 3223-3230. 2011.

https://doi.org/10.1007/s12206-011-0931-9 